\title{
Range and Intensity Vision for Rock-Scene Segmentation
}

\author{
Simphiwe Mkwelo, Frederick Nicolls, and Gerhard de Jager \\ ${ }^{1}$ Defence Peace Security and Safety, \\ Council for Scientific and Industrial Research (CSIR) \\ Meiring Naude road, Lynnwood, Pretoria 0002, South Africa \\ smkwelo@csir.co.za \\ http://www.csir.co.za \\ ${ }^{2}$ Department of Electrical Engineering, University of Cape Town, Rondebosch 7700, \\ South Africa \\ \{fnicolls, gdj\}@dip.ee.uct.ac.za \\ http://www.uct.ac.za
}

\begin{abstract}
This paper presents a methodology for the automatic segmentation of rock-scenes using a combination of range and intensity vision. A major problem in rock scene segmentation is the effect of noise in the form of surface texture and color density variations, which causes spurious segmentations. We show that these problems can be avoided through pre-attentive range image segmentation followed by focused attention to edges. The segmentation process is inspired by the Human Visual System's operation of using a priori knowledge from pre-attentive vision for focused attention detail. The result is good rock detection and boundary accuracy that can be attributed to independence of range data to texture and color density variations, and knowledge driven intensity edge detection respectively. Preliminary results on a limited image dataset are promising.
\end{abstract}

Keywords: surface texture, color density variations, range image segmentation, intensity edge detection.

\section{Introduction}

An instrument for measuring rock fragmentation is invaluable to the mining and mineral processing industries where it can be used to monitor, optimize and control blasting and communition. Image processing in particular, has been widely accepted as the analysis method of choice for the estimation of rock-size because of: the non-intrusive nature of the measurement process; the capability of measuring continuously and in real time; the repeatability of measurements given the same scene and lighting conditions; and the absence of moving mechanical parts which implies little or no maintenance.

Intensity image analysis, in particular, has been a research area of focus for many of the recent academic [1], 2], 3] and operational [4, [5], 6] systems, mainly because the imaging sensor is sensitive to shadows which are formed 
around the individual particles. These shadows are detected and completed as rock outlines during rock scene segmentation. However, limitations of intensity sensors are well documented [7, [2]: the loss of a dimension due to the projective nature of the sensor; and the loss of small particles due to the limitation in the sensors resolution, etc. These limitations introduce errors into the measurement process which are further increased by errors that can be attributed to the environment under imaging: poor lighting; color density and texture variations.

Lighting conditions can be controlled through the elimination of natural lighting and proper design of synthetic lighting [2]. Color density and texture variations define a rock-scene in intensity but do not necessarily correlate to range image data except perhaps at the edges of rocks. High quality range images of rock-scenes have been segmented using 3D morphological techniques 8 for the purposes of rock-size estimation. However, the processing that is required to produce such high quality data inhibits the practical implementation of such a system in a real plant. Consequently, rapidly generated range imagery of sufficient quality can be complemented with corresponding intensity in a multisensor fusion framework.

A review of current trends and future directions in multisensor integration and fusion is presented in 9], where a distinction between multisensor integration and fusion is made. Multisensor Integration is viewed as the general connenctivity of sensors that produce information at system architecture and control level. Fusion, on the other hand, is viewed as the process of combining multisensory information using statistical or mathematical methods. Multisensor integration of infrared and visual sensors has been used for human form detection[10. Range and intensity images have been fused for mobile robot localization in robotics 11 and deformed object identification and bin picking in materials handling[12]. Range maps have been fused with intensity edge maps to segment noisy range images 13 .

We present a methodology that combines the analysis of range and intensity images to address the problem of texture and color density variations during rock edge extraction. Experience and previous work [2] has shown that the human visual system (HVS) is more than capable of rock-scene segmentation and therefore serves as a platform upon which the image analysis is based. Initially, pre-attentive range vision in the form of extracting preliminary rock boundaries from a range image is executed. The boundary information is eventually used as a priori knowledge for focused attention to intensity edges. A description of the pre-attentive range segmentation algorithm is presented in section 2 ; section 3 describes the focused intensity edge detection algorithm; a methodology for performance evaluation is presented in section 4; Results of the experiments are presented in section 5 ; and conclusions are drawn in section 6 .

\section{Pre-attentive Range Vision}

Segmentation of range data is an important part of range image perception and understanding. The general problem of range image segmentation is that of partitioning the range image into disjoint surfaces representing individual 
objects or a single object. In this work, the former condition is encountered, where each surface represents a single rock in the image. The problem has seen many solutions such as the HK map iterative region growing [14, the effective jump-diffusion method [15], scan-line grouping [13], methods using morphological operators [16, 8] and others. In [13, intensity data in the form of Intensity Edge Maps (IEM) are fused with range data to improve range segmentation. In this work, range segmentations are used to improve intensity image segmentation, see section 2.2. We use a global range segmentation technique referred to as rock model extraction, which is based on morphological operators. Specifically, the watershed and distance transforms are used, as shown in figure 1.

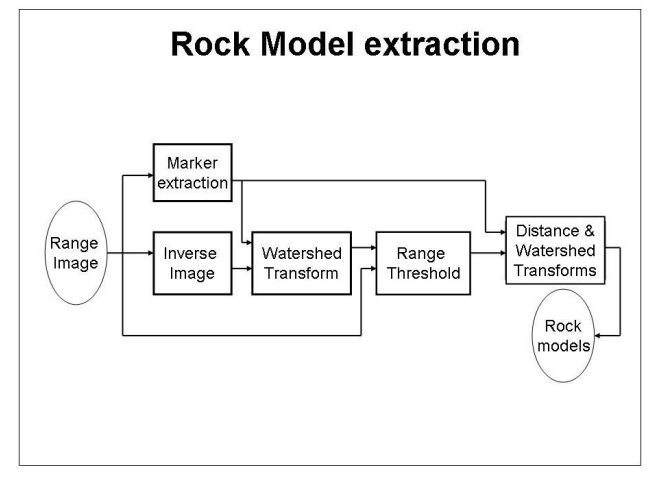

Fig. 1. A block diagram representation of rock model extraction

The processing includes: the estimation of background and foreground range values for range value thresholding to produce a binary image; connected binary models are disconnected using a combination of distance and watershed transforms. As can be seen in figure 2 that the watershed is executed twice for a single range image. The first watershed is used to estimate a range threshold that separates foreground and background range values based on a discrepancy between trough and surface range values. The second watershed combined with the distance transform spatially splits connected binary models that result from the previous process. The distance transform assigns a value for each foreground pixel that is equal to its distance from the background. Consequently, connected binary models will have cone shaped structures whose peaks are centered at the centroids of the models with local minima or saddle points between these peaks. This allows for a watershed transform to create boundaries at the saddle points and hence splitting connected models.

\section{Focused Intensity Vision}

Perception and understanding of intensity data often requires image segmentation. The segmentation of intensity rock-scenes can be difficult without a priori 
knowledge about rocks because of noise, texture and color density variations on rock surfaces. Crida 2] used elliptical models of rocks as a priori knowledge for focused intensity edge detection. He used elliptical probability masks and edge orientations to limit the search for edge pixels. In this work, a similar approach is taken, except a shape model from a range image is used for producing probability masks and edge orientations as shown in figure 2 The process is local by nature and therefore focuses attention to a predefined region-of-interest. Key processing operations in the form of rock edge detection, probability masking and optimization are described in the next section.

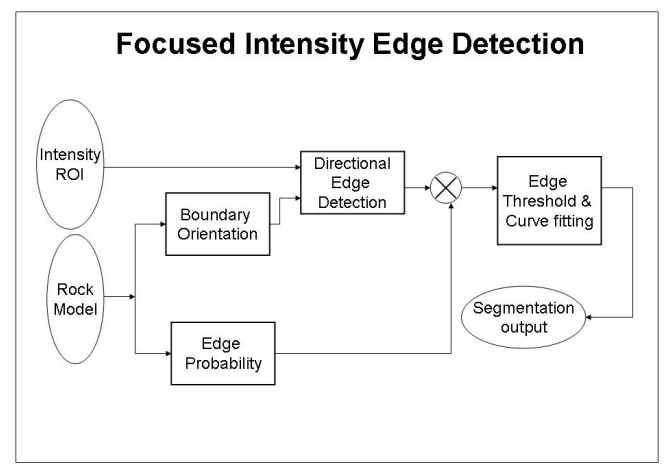

Fig. 2. A block diagram representation of focused intensity edge detection

\subsection{Edge Detection, Masking and Optimization}

The intensity sub-image is subjected to a directional edge detection process that computes edge-responses based on a variable [3-by-3] convolution kernel whose elements depend on the pixel position and orientation of the boundary model at that point. The practical implementation involves predefining a set of possible kernels for a number of arc intervals of a circle which defines the orientation of edges to be detected. The radial convolutions produce noisy edge responses in areas of mismatches between the kernel orientations and edges. We use probability masks to spatially limit the search area of edges of interest. The masks are created by a dilation of boundary models, distance transformation and normalization. The masking is achieved by multiplying the edge response with the probability mask to remove interior texture and color density noise effects. The masked response is then optimized to narrow edge traces through a radial search for pixels with maximum edge responses. Figure 3 shows input intensity, edge response, probability mask, masked response and optimized response sub-images.

\subsection{Rock Shape Extraction}

The objective of this stage is to fit the optimized response by a smooth boundary to extract the underlying rock shape. This involves a transformation from an 


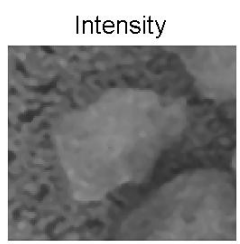

Edge response

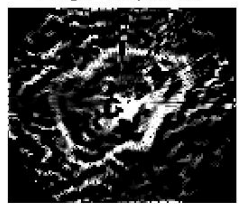

Boundary model

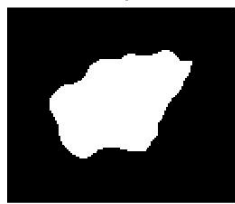

Masked response

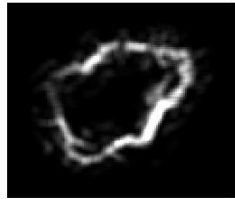

Probability mask

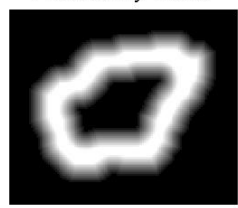

Optimized response

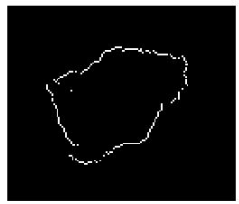

Fig. 3. Image data: intensity, boundary model, probability mask, edge response, masked response and optimized response
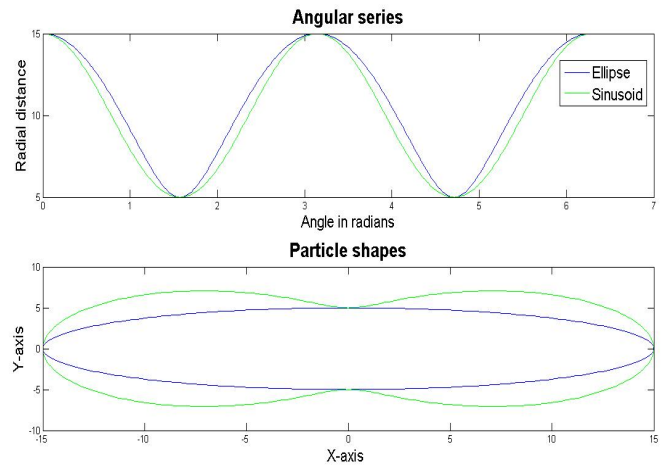

Fig. 4. A sinusoidal signal as an approximation to an elliptical shape

image to an angular-series representation of radial distances. The independent variable becomes angle in radians. The angles range from zero to $2 \pi$ radians and the distance signal wraps around at the zero and $2 \pi$ radians interface. A circle of radius $\mathrm{r}$ in an image becomes a DC signal of $\mathrm{r}$ volts in the angular-series representation. An ellipse becomes an approximately sinusoidal signal with a DC offset equal to the average of the principal components and amplitude equal to the difference in principal components, as shown in figure 4 .

In general, however, ore can consists particles with sharp and pointed edges producing irregular and complex angular-series. We use an $N^{t h}$ order least squares polynomial as a first approximation of the angular-series points and hence extract the underlying shape of the projected rock. The least squares formulation determines coefficients

$$
\mathbf{c}=\left[c_{N}, c_{N-1}, c_{N-2}, \ldots, c_{1}\right]^{T}
$$


from the general vector equation

$$
\mathbf{y}=\mathbf{A c}
$$

as being $\left(\mathbf{A}^{T} \mathbf{A}\right)^{-1} \mathbf{A}^{T}$. This is known as the pseudo-inverse of matrix $\mathbf{A}$. A single row of the matrix $\mathbf{A}$ has the form $\left[x^{N} x^{N-1} x^{N-2} \ldots 1\right]$. The increase in the order of the polynomial will vary the segmentations from simple to complicated models. Least squares is known to perform well provided that there is an effective pre-processing for removing outliers in the data. Because it is not possible to completely remove outliers, it maybe necessary to investigate other curve fitting methods such as Radial Basis functions and robust estimation methods which are known to be less sensitive to outliers.

\section{Methodology}

A laboratory data set in the form of a calibrated stereo pair of consecutive frames of an intensity rock-scene is used to generate a range image using dense stereo reconstruction [17, [18]. Pre-attentive segmentation of the range image, as described in section 2, is applied to produce boundary models, which are used to automatically select image windows of corresponding intensity rocks. Focused intensity segmentation is achieved through using variable edge orientation kernels and probability masks from the boundary models.

The system's performance is evaluated in terms of a visual measure of error from images coupled with numerical errors between automatically extracted

Table 1. The user-input parameter set

\begin{tabular}{|c|c|}
\hline Parameter & value \\
\hline Polynomial orders & 11 and 15 \\
\hline Probability mask width & $60 \%$ of radial distance \\
\hline 2-tail outlier search regions & $50 \%$ of range \\
\hline Intensity Gaussian filter [size, $\sigma]$ & {$[9,0.5]$} \\
\hline
\end{tabular}
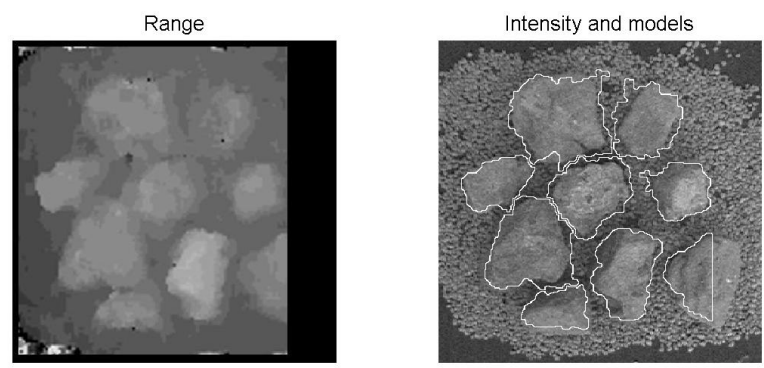

Fig. 5. Experimental data set: the range image and the corresponding reference intensity image overlaid with boundary models 
rock-shapes and hand segmented sub-images. Numerical differences in enclosed areas between the segmentations of the system and the human visual system are computed by image subtraction of the system output from the hand segmented images. The percentage error is computed relative to the HVS segmentations. Table 1 shows the user input parameter set that is used during the experiments and figure 5 is the input data-set to be used by the focused intensity segmentation algorithm.

\section{Results}

This section presents results on a data-set of 8 sub-images. The results are divided into visual and numerical categories based on performances with settings of table 11. The visual data in the form of the input intensity image, extracted model, angular series and segmented rock are shown in figures 6 to 9. Polynomial fitting results with polynomials of order 15 and 11 are shown. The results show, as predicted, that the order 11 polynomial imposes smoothness on the model, while the $15^{\text {th }}$ order can model the intricate complexities of rock shapes. The $15^{\text {th }}$ order polynomial is used for comparisons to hand segmented sub-images.

Table 2 shows the numerical results of a comparison to hand segmented subimages in terms of over-estimation, under-estimation and overall errors. Results show that the worst performance is produced on image 4 of figure [7, with an overall pixel error $25.5 \%$ and an under-estimation component of $24.81 \%$. This image appears not to have clear indications of rock edges of interest. The system performs best on image 5 of figure 8, with a minimum overall error of $4.24 \%$. This image appears to have very good edge information about the rock of interest. Sub-image 3 of figure 7 shows the effect of the sensitivity to outliers of the least squares method. As a result the overall error of $8.59 \%$ has an over-estimation component of $6.55 \%$.

These are preliminary results and therefore it is not reasonable to specify averages and standard deviations as the number of image samples is not sufficient.
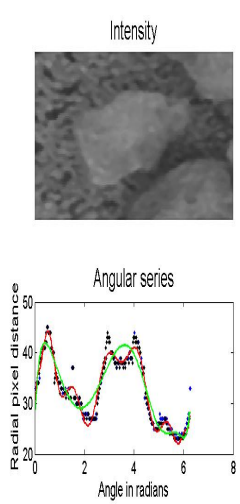
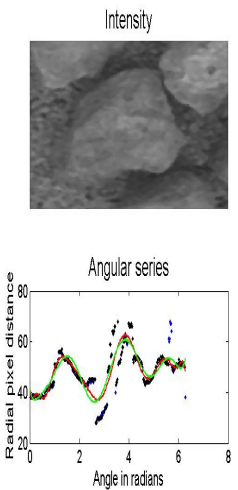
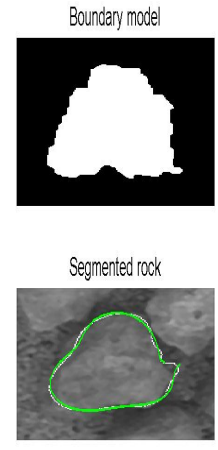

Fig. 6. Rock sub-images 1 and 2 with corresponding results 

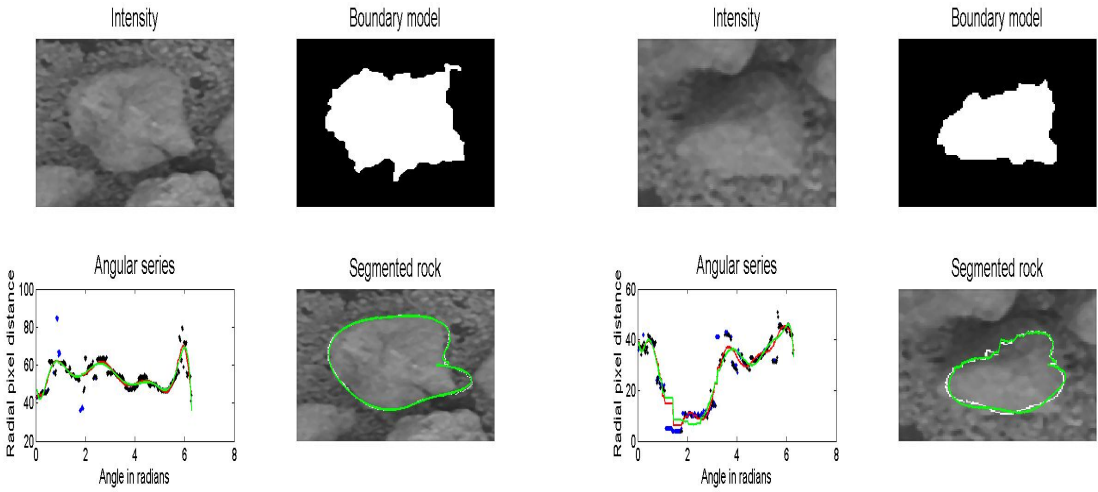

Fig. 7. Rock sub-images 3 and 4 with corresponding results
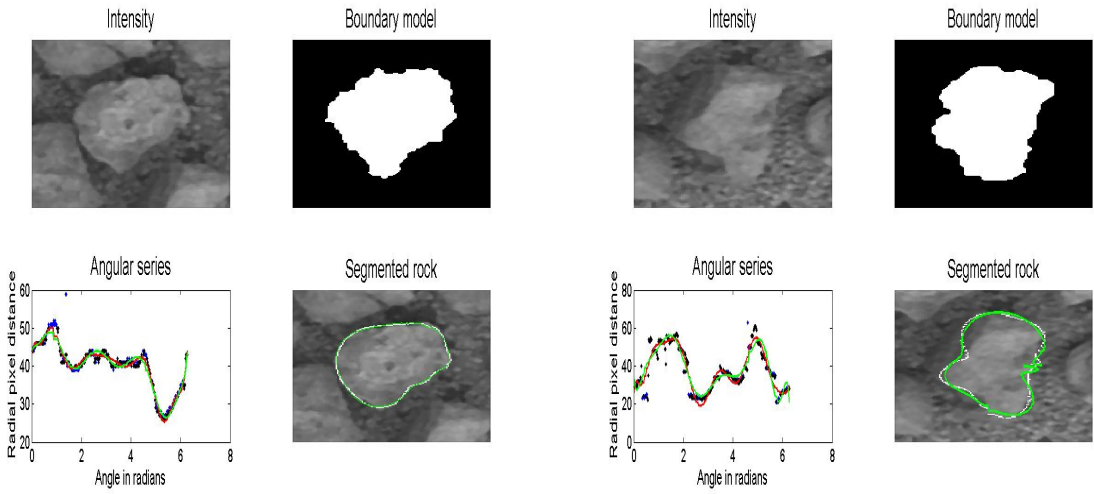

Fig. 8. Rock sub-images 5 and 6 with correpsonding results
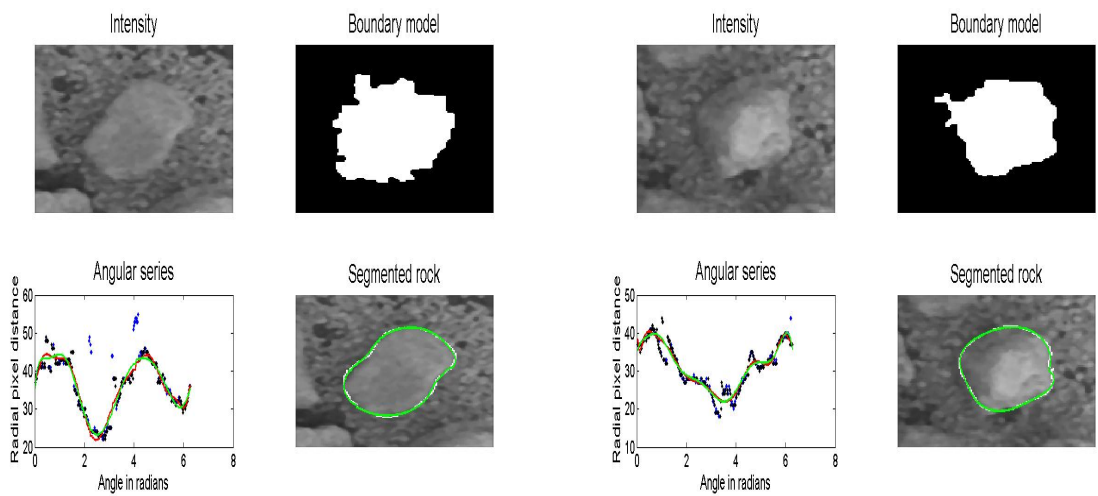

Fig. 9. Rock sub-images 7 and 8 with corresponding results 
Table 2. Segmentation error with respect to HVS segmentation results

\begin{tabular}{|c|c|c|c|}
\hline Image number & under-estimation error & over-estimation error & overall error \\
\hline image 1 & $1.85 \%$ & $4.09 \%$ & $5.93 \%$ \\
\hline image 2 & $9.95 \%$ & $2.96 \%$ & $12.9 \%$ \\
\hline image 3 & $2.04 \%$ & $6.55 \%$ & $8.59 \%$ \\
\hline image 4 & $24.81 \%$ & $0.24 \%$ & $25.5 \%$ \\
\hline image 5 & $1.07 \%$ & $3.17 \%$ & $4.24 \%$ \\
\hline image 6 & $18.83 \%$ & $1.82 \%$ & $20.65 \%$ \\
\hline image 7 & $0.92 \%$ & $4.92 \%$ & $5.84 \%$ \\
\hline image 8 & $5.14 \%$ & $1.66 \%$ & $6.80 \%$ \\
\hline
\end{tabular}

\section{Conclusions}

Based on the above findings and results, the following conclusions can be drawn.

- An HVS inspired methodology for rock-scene segmentation that combines intensity and range image analysis to avoid the effects of texture and color density variations is presented.

- Post-processing in the form of angular series representation and polynomial fitting is used to extract the underlying rock shape with good accuracy. However, visual and numerical results seem to agree with literature that the least squares estimator is sensitive to outliers and therefore other estimators must be investigated.

- The system appears to perform very well on the experimental data set. However, more data with variation in texture and color densities is required for a more rigorous evaluation.

Acknowledgments. The author is grateful to the CSIR Defence Peace Safety and Security, NRF and Anglo-Platinum through MPRU for financial support.

\section{References}

1. Lange, T.B: Measurement of the size distribution of rocks on a conveyor belt using machine vision, $\mathrm{PhD}$ thesis at the University of Witwatersrand (1990)

2. Crida, R.C.: A machine vision approach to rock fragmentation, PhD thesis at the University of Cape Town (1995)

3. Mkwelo, S., De Jager, G., Nicolls, F.: Watershed-based Segmentation of Rock Scenes and Proximitybased Classification of Watershed Regions Under Uncontrolled Lighting. SAIEE Transactions, Research Journal of SAIEE 96(1), 28-34 (2005)

4. Sudhakar, J., Adhikari, G.R., Gupta, R.N.: Comparison of Fragmentation Measurements by Photographic and Image Analysis Techniques. Rock Mechanics and Rock Engineering 39(2), 159-168 (2005)

5. Kemeny, J.: The Split-Online Fragmentation Analysis System (February 12, 2006), ww. .spliteng.com/splitonline/fragmentation.asp 
6. Franklin, J.: Granulometry Analysis Software (February 12, 2006), Www.wipware.com/wipfrag.php

7. Maerz, N.H., Zhou, W.: Optical Digital Fragmentation Measuring SystemsInherent Sources of Error. FRAGBLAST The International Journal for Blasting and Fragmentation 2(4), 415-431 (1998)

8. Thurley, M.J: Three Dimensional Data Analysis for Separation and Sizing of Rock Piles in Mining. PhD Thesis, Monash University, Department Electrical and Computer Systems Engineering (2002)

9. Luo, R.C., Yih, C.C., Su, K.L.: Multisensor fusion and integration: Approaches, Applications and Future Directions. IEEE Sensors Journal 2(2) (2002)

10. Shah, S., Argarwal, J.K., Eledah, J., Ghosh, J.: Multisensor Integration for scene classification: An experiment in human form detection. In: International Conference on Image Processing, Santa Barbara, October 26-29, 1997 (1997)

11. Neira, J., Tardos, J.D., Horn, J., Schmidt, G.: Fusing range an intensity images for mobile robot localization. IEEE Transactions on robotics and automation 15(1) (1999)

12. Umeda, K., Arai, K.: Industrial vision system by fusing range and intensity image. In: Proc. of IEEE Int. Conf. on Multisensor fusion and integration for Intelligent Systems, October 2-5, 1994 (1994)

13. Zhang, Y., Sun, Y., Sari-Sarraf, H., Aidi, M.A.: Imapact of Intensity Edge Map on Segmentation of noisy range images. In: Proc. of Spie Conf. on Three Dimensional Capture and Applications III, San Jose,CA, vol. 3958, pp. 260-269 (2000)

14. Besl, P.J., Jain, R.C.: Segmentation Through Variable Order Surface Fitting. IEEE Trans. on Pattern Analysis and Machine Intelligence, PAMI 10(2), 167-192 (1988)

15. Han, F., Tu, Z., Zhu, S.: Range image segmentation by an effective jump diffusion method. IEEE Transactions on pattern analysis and machine intelligence 26(9) (2004)

16. Gee, L.A, Abidi, M.A.: Segmentation of range images using morphological operations: Review and examples. In: SPIE Conference on Intelligent Robots and Computer Vision XIV, Philadelphia, PA, vol. 2588, pp. 734-746 (October 1995)

17. Mkwelo, S., De Jager, G., Nicolls, F.: Three Dimensional rock-scene modelling using dense stereo reconstruction. In: 13th Proceedings of PRASA (November 2006)

18. Scharstein, D., Szeliski, R.: A taxonomy and evaluation of dense two frame stereo correspondence algorithms. International Journal of Computer Vision 47(1), 7-42 (2002) 